\title{
TRANS-SULCAL MICROSURGICAL APPROACH TO INTRACEREBRAL LESIONS
}

\author{
St. Valkanov*, P. Valkanov, M. Kirkov \\ Clinic of Neurosurgery at Stara Zagora University Hospital, Trakia University, Stara Zagora, Bulgaria
}

\begin{abstract}
Objective: The sophistication of medical technologies facilitates the diagnosis of various lesions in the deep parts of the brain hemispheres. That occurs the need of improved surgical techniques that these lesions can be achieved with minimal damage to the cortex. Our goal was to introduce into clinical practice trans-sulcal microsurgical approach to intracerebral lesions. 287 patients with intracerebral lesions were operated for the period of 1995-2012. Of these 142 cases of glial tumors and 61 metastases located supratentorialy, 21 cases of cerebellar tumors and 63 cases of hypertensive intracerebral hematoma. Methods: To minimize iatrogenic damage of the cerebral cortex, the trans-sulcal microsurgical approach was used in all of these cases. Results: The approach was successfully applied in all 287 cases. In 271 of them there was a regress in neurological deficit and improvement of the overall condition in the early postoperative period. In 16 of cases there was a lethal end due to accompanying severe somatic disorders. Conclusions: Trans-sulcal approach ensures atraumatic microsurgical access to intracerebral lesions, preserving as much as possible the physiological and anatomical structures of the brain. The surgical intervention creates the least amount of discomfort for the patient and the recovery post-surgical period is fast and with minimum complications.
\end{abstract}

Key words: trans-sulcal approach, trans-sylvian approach, intracerebral lesions

\section{INTRODUCTION}

With the introduction of microsurgery more than 40 years ago, the angiography and other invasive techniques such as pneumoencephalografy, ventriculography were used in preoperative imaging of intracranial lesions, since at that time the most commonly used modern methods such as CT, MRI, positron emission tomography (PET) and superselectiv catetherized angiography did not exist. These contemporary, less aggressive and invasive but more accurate diagnostic methods can accurately depict pathologic and patomorphological characteristics of the lesion (1).

The development of medical technologies allows the diagnosis of small asymptomatic lesions located in the deep parts of the brain hemispheres. So there appears a need of

*Correspondence to: St. Valkanov, Clinic of Neurosurgery, University Hospital, Stara Zagora, Bulgaria,nhk_stz@yahoo.com improved surgical techniques with which these lesions can be achieved with minimal damage to the cortex. Trans-sulcal microsurgical approach is based on the idea that the less is the damage to the cerebral cortex and white matter, the less damage is caused to the patient by the surgeon (2). In 1986, one of the pioneers of microneurosurgery - professor M.GaziYasargil used and popularised the idea of approaching lesions that were previously considered as "unaccessible" by using subarachnoid system surrounding the brain.

He demonstrates that the natural anatomical pathways (cysternal, sulcal, fissural, ventricular) can be used to access the deeply situated tumors and to make a clean "tumorectomy" thus avoiding the compression or resection of normal neural tissues (3).

There's no systematic study in Bulgarian literature on the applying of microsurgical transsulcal approach in the surgical treatment of intracerebral lesions. This stimulates us to aim at 
exploring and applying the trans-sulcal microsurgical approach in the surgical treatment of intracerebral lesions in the specific conditions of our clinic.
VALKANOV ST., et al.

\section{MATERIALS AND METHOD}

In this study, we analyze clinical and operative results of 287 patients with intracerebral lesions (Tables 1 and 2), treated surgically by transsulcal microsurgical approach at the Neurosurgical Clinic of Stara Zagora University Hospital for a period from 1995 to 2012.

Table 1. Structure of the operated patients with intracerebral lesions by nozolological units.

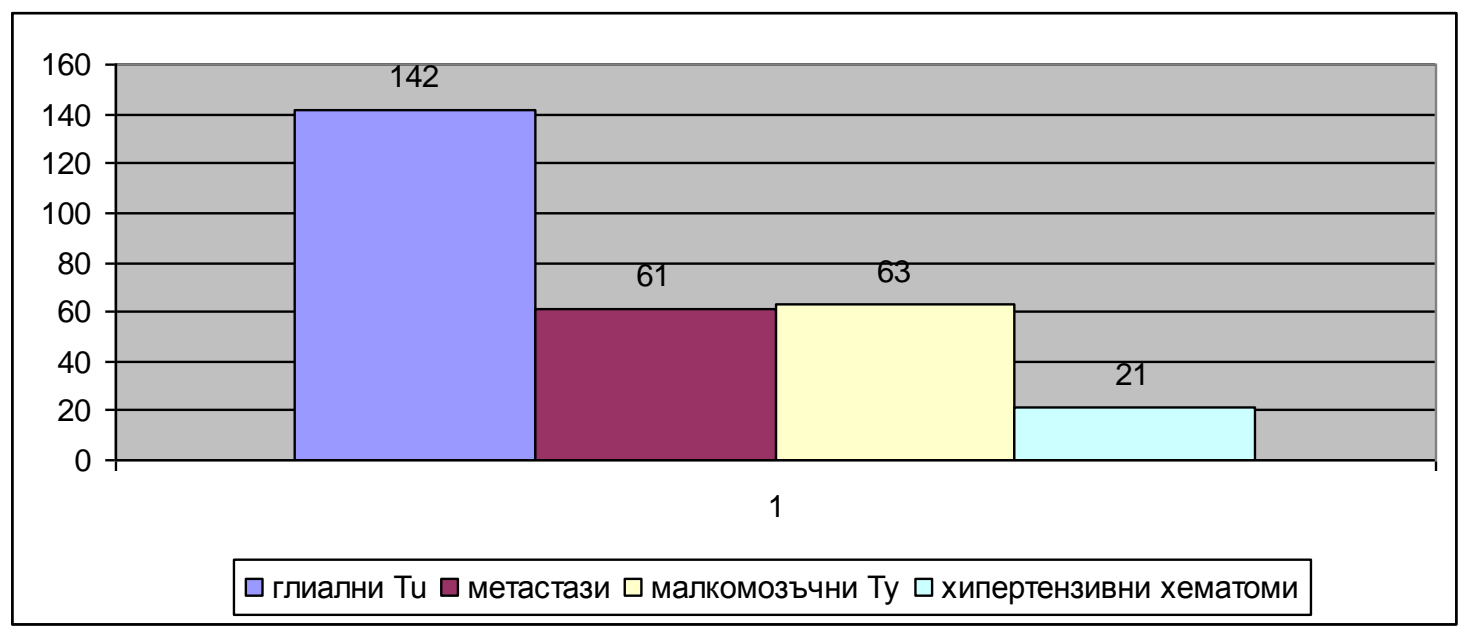

Table 2. Structure of the operated patients with intracerebral lesions by age.

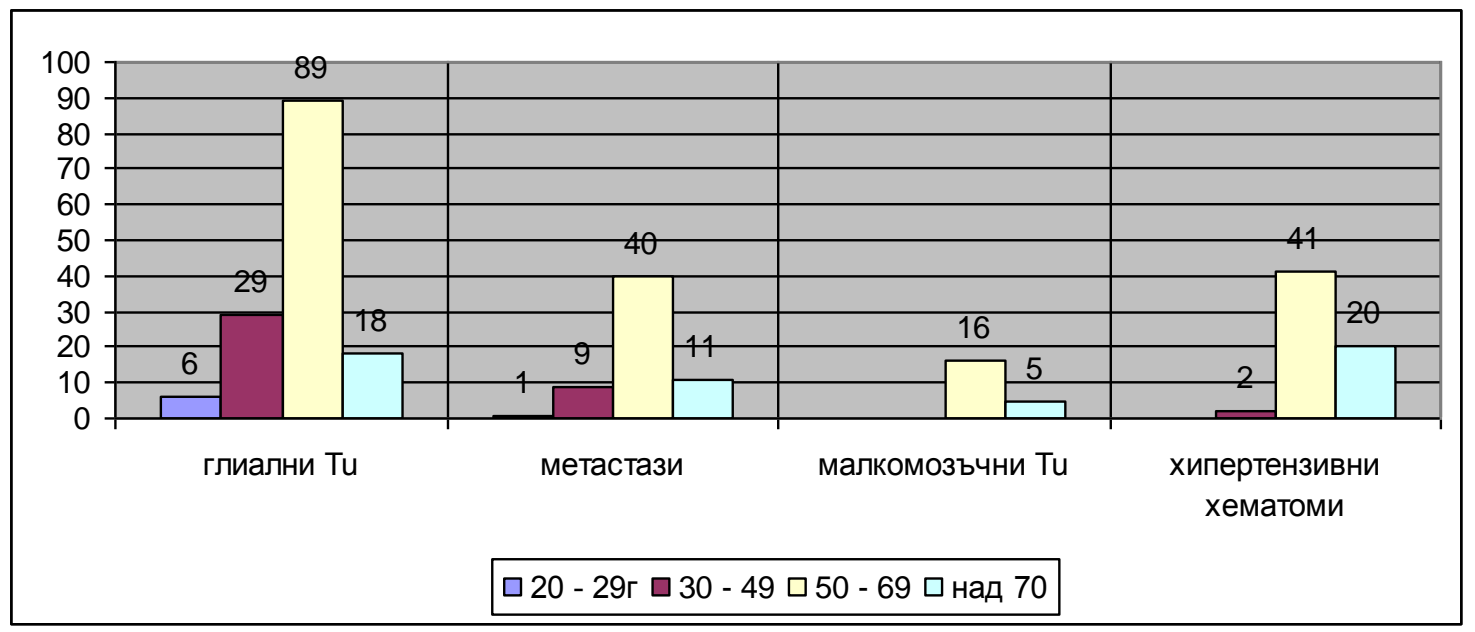

It is preferable to choose a sulcus in which there are no large venous channels, but sometimes it is impossible to avoid them. The presence of a large vein in the sulcus is by no means absolute contraindication for the trans-sulcal approach. The brain must be relaxed, as it is very difficult to dissolve the sulcus when the brain is tense and actually that causes more damage to the cortex than when a small cortical incision is made.

The adequate relaxation, proper ventilation, as well as the application of Mannitol (i.v.) helps to achieve that. The operative field must be kept moist as it is much easier to desect the arahnoid when tissues are moist. The arachnoid layer overlying the sulcus is incised by an arachnoid knife in an area without underlying blood vessels (Figure 1A)

The overlying along the sulcus arachnoid layer is cut to the long axis of the sulcus using microscissors (Figure 1B). Usually after the release of CSF, the superficial arachnoid layer tends to fall in the sulcus. If there is a large artery or vein on the surface, the arachnoidal layer is opened on one side of the vessel so to be covered and 
protected by it. The opening of the sulcus should be extended to the end of the sulcus - where it meets the adjacent sulci.

Once the surface arachnoid layer is open wide, the walls of the sulcus is carefully dissolved by blunt dissection with the arms of the bipolar forceps and the aspirator, which provides a sufficient separation of the sulcal walls (Figure 1C, D).
The arachnoid strands that crossing sulcal space are cut then. It should be emphasized that it is necessary to do their utmost to preserve even the small branches of arteries or venules passing through the sulcus. This is important because some of these vessels didn't end in the sulcal walls, but only intersect and continue to supply another area.

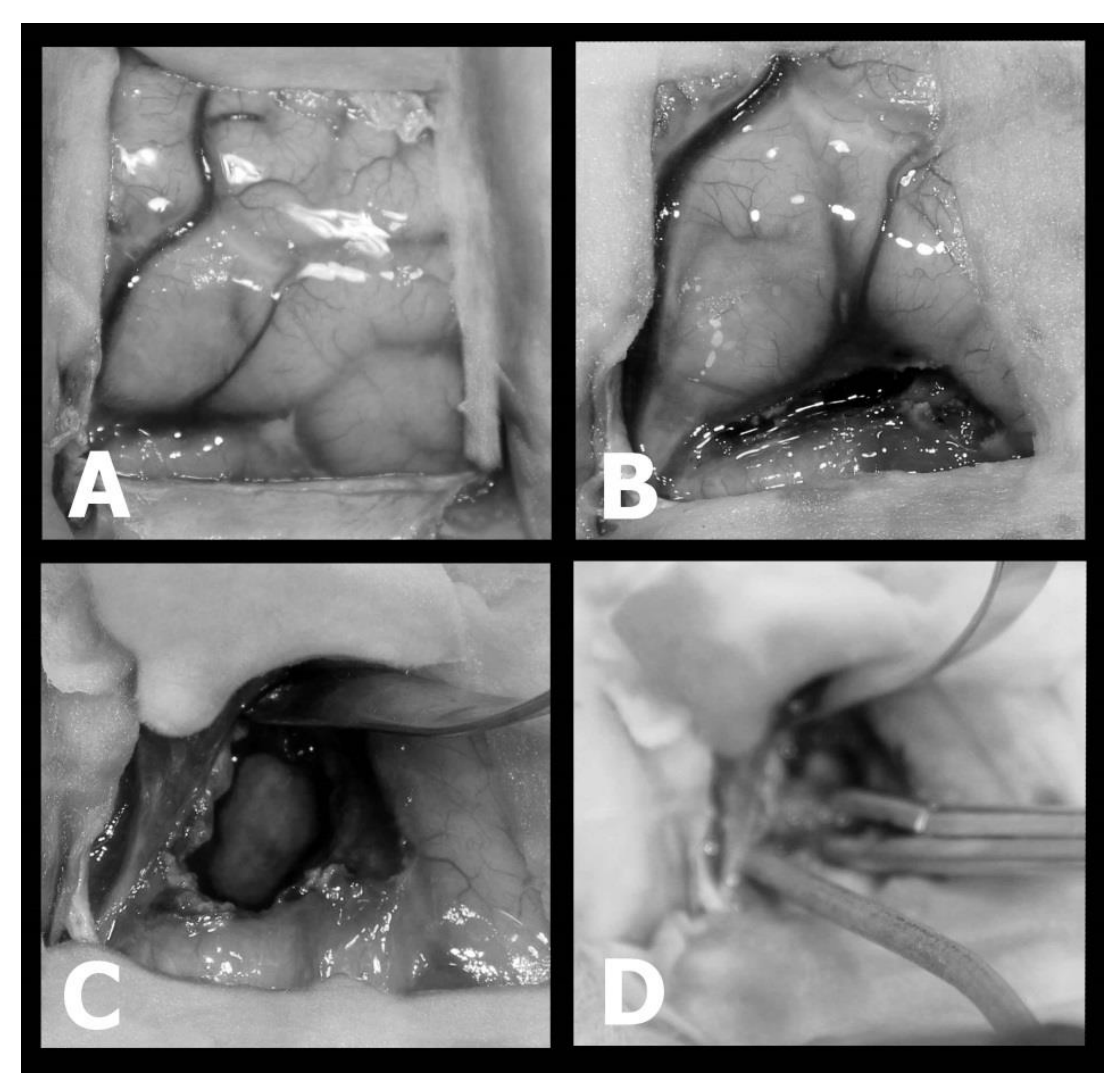

Figure 1. Using of trans-sulcal microsurgical approach to intracerebral lesions.

We used trans-sylvian approach, first described by Suzuki and Sato 1976 in the surgical treatment of hypertensive putamenal hematomas. The technique involves classical pterional craniotomy for which the projection of the Sylvan fissure is in the center of the surgical area (Figure 2A). The dissection of the Sylvan fissure continues under optical enhancement, laterally from the Sylvan vein (Figure 2B) in the external chamber of the Sylvian tank, while protecting as much as possible the venous inflows and the branches of the midbrain artery. The length of the dissection must be 3-4 $\mathrm{cm}$ for a better identification of the insular cortex. Using a ventricular needle we verified the hematoma (Figure 2C) and after incision of the insular cortex we penetrated in its cavity and evacuated it by aspiration and lavage with physiological serum (Figure 2D). Following thorough hemostasis, we restored the cerebral membranes, performed epidural pipe drainage and restoration of the bones, muscles and skin.

\section{RESULTS AND DISCUSSION:}

In 271 of 287 operated patients there was a regress of the neurological deficit and improvement of the overall condition in the early post-surgical period (Table 3). This allows to guide the patients with brain tumors and metastases to continue the complex treatment in local oncologic centers, and those with intracerebral hematoma to be covered in the outpatient dispensary Health Network to conduct rehabilitation procedures. 


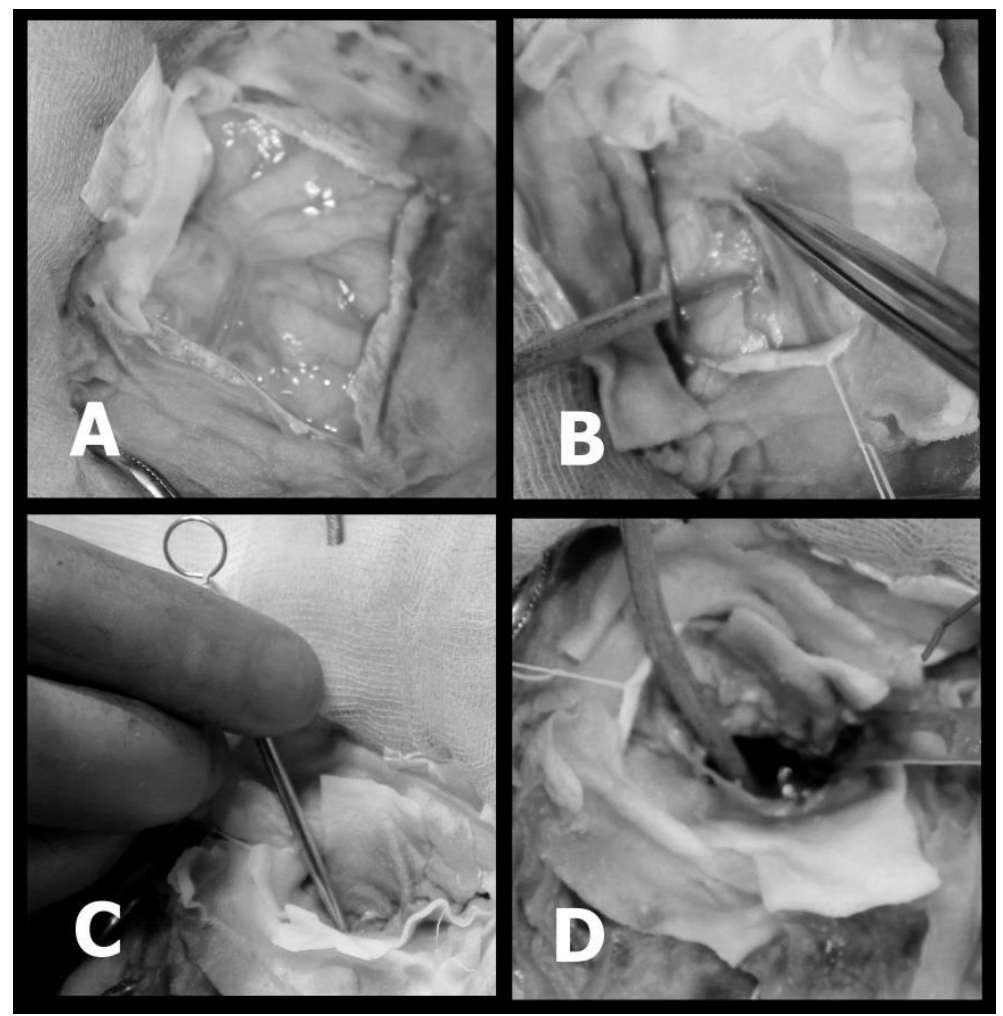

Figure 2. Using of trans-sylvian approach to hypertensive putamenal haematoma.

Table 3. Operating results in 287 patients with intracerebral lesions

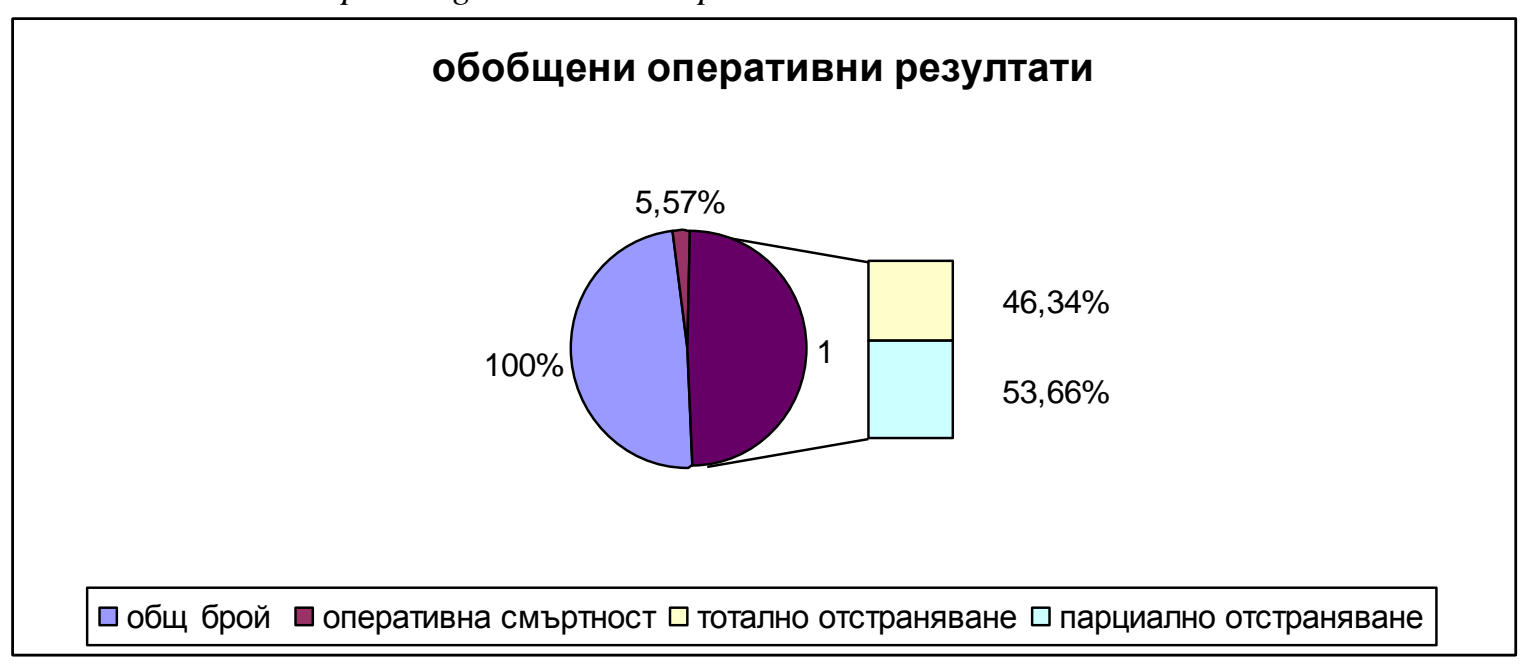

The analysis of the remaining 16 patients who had a lethal outcome showed that their condition was aggravated by preoperative concomitant somatic diseases and superimposed postoperative complications of the respiratory and cardiovascular systems which led to lethal end (Table 4).

The presented clinical-operative data indicates that the surgical resection of intracerebral lesions and particularly those in the dominant hemisphere is often a challenge. The standard approaches required a significant retraction of brain structures or resection of normal tissue.

The trans-sulcal microsurgical approach is minimally invasive technique, which is relatively simple and provides added accuracy and safety to the surgical procedure (4) (Figures 3 and 4). It should be recommended especially when working in the dominant brain hemisphere, to 
VALKANOV ST., et al.

avoid disturbances in higher cognitive functions. Using it requires in-depth knowledge of the opperative area mycroanatomy, and knowledge

Table 4. Early postoperative complications. of the existing anatomical markers for selecting an appropriate sulcus to access the lesion $(5,6$, $7,8)$.

\begin{tabular}{|l|c|l|}
\hline \multirow{2}{*}{ Neurological } & Early postoperative complications. & \\
& Stage changes in consciousness- 16 & $5,57 \%$ \\
\cline { 2 - 3 } & Aggravation of neurological symptoms- 10 & $3,48 \%$ \\
\hline Inflammatory & Operative wound infections- 7 & $2,44 \%$ \\
\cline { 2 - 3 } & bronchopneumonia - 15 & $5,23 \%$ \\
\cline { 2 - 3 } & Urinary tract infection-5 & $1,74 \%$ \\
\hline $\begin{array}{l}\text { Identified on the } \\
\text { postoperative CT }\end{array}$ & Blood in the operative field - 40 & $13,94 \%$ \\
\hline
\end{tabular}
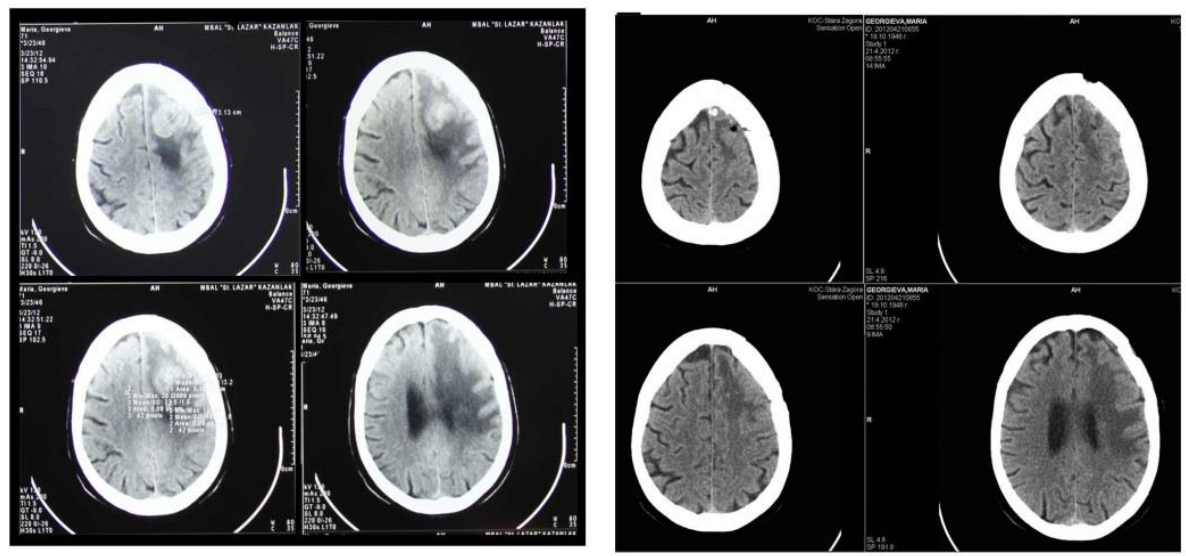

Figure 3. Pre- and postoperative CT in a patient with metastasis.
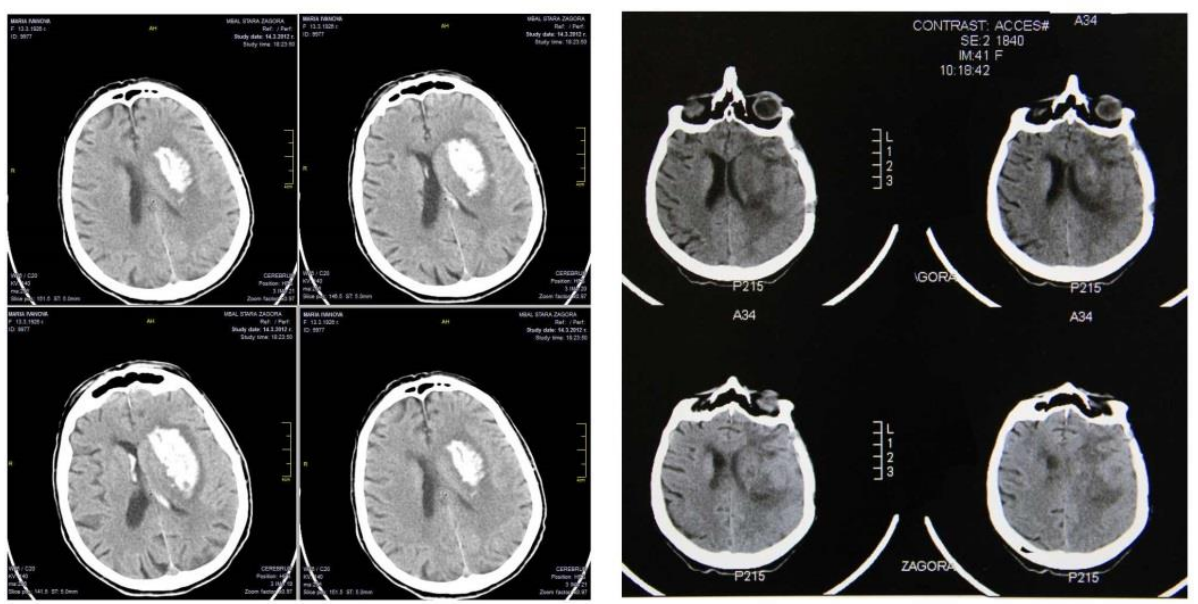

Figure 4. Pre- and postoperative $\mathrm{CT}$ in a patient with hypertensive putamenal haematoma. 


\section{CONCLUSIONS}

1. The trans-sulcal microsurgical approach ensures less traumatic surgical interventions.

2. Preserves as much as possible the physiological and anatomical structures of the cerebrum.

3. The surgical intervention creates the least amount of discomfort for the patient.

4. The recovery post-surgical period is fast and with minimum complications.

\section{REFERENCES}

1. A. Perneczky, W. Muller-Forell, E. van Lindert, G. Fries. "Keyhole concept in Neurosurgery", Thieme, New York, p 4, 1999.

2. Ravi Ramamurthi, K. Sridhar „Trans-sulcal Approach to Intracerebral Lesions", Textbook of Operative Neurosurgery, vol.2, chapter 38, 335-337

3. Yashargil M.G. "Microneurosurgery of CNS tumors". IV, B, New York: Thieme Medical Publishers Inc, p 91, 1996.
VALKANOV ST., et al.

4. A. Jabre, A. Patel. Transsulcal microsurgical approach for subcortical small brain lesions: technical note. Surgical Neurology 65312 314, 2006.

5. Mikuni N, Hashimoto N. A minimally invasive transsulcal approach to the paracentral inner lesion. Minim Invasive Neurosurg. Oct;49(5):291-5, 2006.

6. Yasargil MG. Microneurosurgery: volume 3B. Surgical concerns. New York 7 Thieme; p. 25- 53, 1988.

7. Oka, H.,Kawashima, M.,Shimizu, S.,Utsuki, S.,Osawa, S.,Sato, K.,Fujii, K.,Rhoton Jr., A.L. Microsurgical anatomy of the lateral ventricle and surgical approaches. Japanese Journal of Neurosurgery, Volume 20, Issue 6, Pages 418-423, 2011.

8. Germano IM, M.D. Transsulcal approach to mesiotemporal lesions. Anatomy, technique, and report of three cases. Neurosurgical focus, 5: 1-8, 1996. 